\title{
La automatización de la industria norteamericana en el contexto de la competencia internacional
}

\author{
Alejandro Mungaray Lagarda \\ Universidad Autónoma de Baja California
}

\begin{abstract}
Resumen
El propósito central de este trabajo consiste en presentar algunas ideas en torno a la automatización que actualmente se viene desarrollando tan intensamente en algunas de las ramas claves de la economía industrial norteamericana. Sin embargo, no se pretende que tal ordenamiento consista tan sólo en la descripción de la evolución de algunos indicadores que reflejarán el proceso de creciente automatización en dichos sectores, más bien se trata de ordenar la información de que se dispone para ilustrar este fenómeno, en torno a un marco conceptual de mínima elaboración que proporcione una explicación, o un porqué de éste. En la medida en que se retarde la implementación de nuevas formas de producción, se incrementarán las posibilidades de una respuesta de la población trabajadora, tendiente a modificar la estructura de las relaciones sociales de producción capitalistas.
\end{abstract}

Palabras clave: automatización, mercado internacional, competencia, crisis, reordenamiento competitivo.

\section{Astract}

The main objective of this work is to orderly present certain ideas about automation process that has been intensely developed at some key areas of the American industry. However, this project will not only describe the evolution of some indicators that show the growing automation process on these sectors, but to classify this information that illustrate this phenomenon around a minimal conceptual frame that proposes an explanation, or at least the reason for this. As far as this measure delays the implementation of new forms of production, the possibilities of getting answers from the working class will be increased, in order to modify the structure of the social relations of a capitalist production.

Keywords: automation, international market, competence, crisis, competitive reordering. 


\title{
LA AUTOMATIZACION DE LA INDUSTRIA NORTEAMERICANA EN EL CONTEXTO DE COMPETENCIA INTERNACIONAL*
}

\author{
Por \\ Alejandro Mungaray Lagarda**
}

\section{INTRODUCCION}

El propósito central de este trabajo consiste en presentar con un cierto orden, algunas ideas en torno a la automatización que actualmente se viene desarrollando tan intensamente en algunas de las ramas claves de la economía industrial norteamericana. Sin embargo, no se pretende que tal ordenamiento consista tan sólo en la descripción de la evolución de algunos indicadores que reflejaran el proceso de creciente automatización en dichos sectores. Más bien se trata de ordenar la información de que se dispone para ilustrar este fenómeno, en torno a un marco conceptual de mínima elaboración que proporcione una explicación, o si acaso un intento, del porque de éste.

Un elemento de tipo metodológico que sirve como punto de partida en este trabajo, es la consideración del desarrollo de la "Estructura técnicoproductiva" de la sociedad, que se refiere a la forma que adopta el proceso de trabajo en términos de la relación entre hombres y medios de producción, en estrecha relación con los aspectos que influyen en su desarrollo económico, político y social ${ }^{1}$.

Ahora bien, esta consideración obliga a colocar la automatización de la producción en el marco de un proceso histórico de evolución de las formas de apropiación del plusvalor como fuente de la ganancia. Estas formas, a su vez, deben ubicarse como respuestas internas a nivel del capitalista individual, para dar salida al problema de la reducción de la productividad en un contexto competitivo. Por tal razón, el intento explicativo del fenómeno de la automatizacion de la producción, se emprende desde la perspectiva de la crisis de la tasa de ganancia en determinadas ramas de la industria norteamericana debido al descenso, estancamiento y/o menor crecimiento de la productividad del trabajo asalariado, que disminuye el monto de plusva-

\footnotetext{
*Ponencia presentada al V Encuentro Internacional de Ingeniería Industrial, organizado por el Instituto Tecnológico de Cd. Juárez, Chihuahua, 26 al 30 de septiembre de 1983.

**Director del Instituto de Investigaciones Económicas y Sociales de la Universidad Autónoma de Baja California, en Tijuana

${ }^{1}$ Este concepto es elaborado por Marx para la época moderna a partir de las hipótesis que sobre las diversas fases del desarrollo de la economía había desarrollado la escuela histórica de la economía. Ver Bolchini (1980):28.
} 
lor generado aumentando el valor de las mercancías haciéndolas menos competitivas y eI valor de la fuerza de trabajo que por tanto se reproduce en un tiempo mayor de la jornada de trabajo ${ }^{2}$.

Con esto se quiere decir que dentro del desarrollo del capitalismo, y dado un contexto de competencia permanente, la "estructura técnicoproductiva" de los capitales individuales tiende a agotar las posibilidades de generar más plusvalor y por lo tanto a declinar su potencialidad como elemento competitivo. Esto se resuelve mediante la transformación de la "estructura técnico-productiva" por otra que, dadas ciertas características innovadoras, permita generar mayor volúmen de plusvalor, vale decir, de ampliar la brecha entre el tiempo de trabajo socialmente necesario para la reproducción del valor de la fuerza de trabajo y el tiempo de trabajo excedente de la misma apropiado por el empresario.

De esta forma, la automatización de la actividad productiva aparece como una respuesta capitalista dada por capitalistas individuales en permanente competencia por salir de sus crisis individual, que es a la vez una crisis global del capitalismo, manteniendo posiciones competitivas favorables en un nuevo contexto de competencia oligopólica, resultante de un proceso de concentración y centralización de los capitales que se hace más fuerte durante la crisis. Esto se Iogra revolucionando las condiciones de producción, esto es, el proceso de trabajo, de manera tal que se posibilite una mayor explotación de la fuerza de trabajo en términos de una mayor productividad medida por una mayor producción de plusvalor relativo y por el descenso del valor unitario de las mercancías.

Con lo anterior se quiere reafirmar la siguiente idea: en un momento dado del desarrollo de la producción social y con un nivel de técnica dado, los niveles de explotación de la fuerza de trabajo o la productividad del mismo, llegan a un límite social que sólo puede ser abatido mediante modificaciones sustanciales en el proceso de trabajo y/o en los elementos que lo integran, a través de la modificación de la "estructura técnico-productiva". Esto, por supuesto, no ocurre aisladamente. Trae consigo cambios sustanciales en el nivel de empleo, en la jornada de trabajo, en la conformación de diferentes sectores sociales, etc.

Estas líneas tienen un carácter provisional y hasta cierto punto superficial, por cuanto que lo que hay que revisar para el entendimiento de la crisis y las modificaciones que en virtud de ella ocurren en los procesos trabajo, requieren no sólo de la revisión permanente y actualizada de la producción

2Para los criterios metodológicos utilizados en el estudio del agotamiento de la "estructura técnicoproductiva", ver Marx (1978) T. 111:213 y Agietta (1979): 99. 
teórica que interpreta el fenómeno y de las metodologías con que se elabora la información de tipo empirico que muestra el desarrollo concreto de la misma. Requiere además de la revision de los propios esquemas de interpretación y saber reconocer sus alcances y/o limites en aras de un conocimiento lo más objetivo posible de la situación concreta que sin embargo, nunca es del todo absoluto. En otras palabras, en una realidad dialéctica, en movimiento y cambiante, es menester medir permanentemente el grado de validez de nuestro marco analítico, asi como los alcances de éste.

\section{CRISIS Y REORDENAMIENTO COMPETITIVO}

El proceso de producción capitalista se desarrolla en un contexto sumamente contradictorio, caracterizado en lo fundamental y en lo general por una confrontación constante entre el capital y el trabajo. Weisskopf demuestra en un trabajo reciente. que la caida de la tasa de ganancia de la industria manufacturera norteamericana entre 1949 y 1975 , se explica más que nada por cl creciente poder de negociación de la fuerza de trabajo ${ }^{3}$. Luego entonces no extraña que los métodos de transformación de la estructura productiva cstén encaminados a eliminar fuerza de trabajo y minar fuerza de trabajo y minar con ello el poder de negociación global de los sindicatos. Sin embargo, el contexto de competencia intercapitalista impide que el desarrollo de la capacidad productiva del trabajo a través de la modificación de la "estructura técnico-productiva", se realice mediante un esfuerzo planificado ${ }^{4}$ En efecto, la resolución al problema de la tasa de ganancia en descenso por el agotamiento de la "estructura técnico-productiva", ocurre con la transformacion de dicha estructura por otra que posibilite una mayor productividad de la fuerza de trabajo $y$, por tanto, un mayor plusvalor. Esto implica un reordenamiento social de la estructura productiva y de los sectores sociales en una situación diferente a la planteada originalmente.

Ahora bien, este reordenamiento social de la estructura productiva no es otra cosa que el reordenamiento competitivo, por que la competencia intercapitalista y el progreso tecnológico son elementos que sc impulsan reciprocamente. Así, en un marco de creciente competencia e innovación tecnológica es obvio suponer que el proceso de innovación lleve a que algu na de las empresas sc coloque, aunque sea mientras la innovación no se difunda plenamente, con ventajas sobre otras en términos de una ganancia extraordinaria obtenida al vender por encima de su costo de producción, pero por abajo de los precios de mercado en que se ubiquen las demás empresas del ramo.

Esto le permitirá un mayor nivel de acumulación en la medida que esa ganancia sea reinvertida productivamente, y por tanto, un mayor crecimiento

\footnotetext{
3Weisskopf (1980):100-104.

${ }^{4}$ Castells $(1981): 103$,
} 
que otras empresas. Luego entonces, los diferentes ritmos de acumulación de las empresas provocan los crecimientos desiguales de las mismas. Este crecimiento, que no es otra cosa que la acumulación creciente de tecnología productiva en detrimento de la fuerza de trabajo empleada para la transformación industrial de los productos mediante su reunión en un mismo proceso productivo, es lo que aqui se entiende como proceso de concentración de capital.

El agudizamiento de la competencia, el agotamiento de la "estructura técnico-productiva", la caída de la ganancia y la imposibilidad de algunos empresarios para renovar su estructura tecnológica, ayuda a profundizar más los diferenciales de productividad entre grandes y pequeños de la misma ra$\mathrm{ma}, \mathrm{y}$ por tanto, sus diferenciales de ganancias y posibilidades de innovación tecnológica. En consecuencia, tiende a suceder que los más pequeños y débiles financieramente quiebren o sean absorvidos por los más grandes y fuertes, y además que estos se desplacen de una esfera de actividad a otra, integrando verticalmente su actividad empresarial. En otras palabras, la crisis, a la vez que recesión de la actividad productiva, significa un mecanismo de restructuración de la industria en nuevos niveles competitivos mediante la eliminación de la ineficiencia productiva y la integración vertical y horizontal de la producción. Lo primero se manifiesta en lo que se conoce como quiebras y lo segundo en lo que se conoce como centralización o absorción de capitales. ${ }^{5}$

La crisis de la ganancia ocurrida por el agotamiento de la capacidad productiva de la industria norteamericana se profundizó a finales de los setenta. Sin embargo, aunque fue la economía norteamericana la que primero resintió Ia situación, para principios de los ochenta ya se había generalizado a todos los países con alto desarrollo industrial y es en las industrias más avanzadas de estos donde se empiezan a realizar los recambios tecnológicos necesarios para conformar una nueva "estructura técnico-productiva" basada en la automatización.

La mejor prueba de la restructuración competitiva del capitalismo en Estados Unidos y en los más importantes países industrializados, la constituye el proceso de quiebras que no significa otra cosa que la eliminación de la ineficiencia competitiva. Entre los últimos años de la década pasada y los primeros de la presente, el número de quiebras ha ido en aumento en tanto que el de fusiones en descenso. En efecto, en Estados Unidos el número de quiebras individuales entre 1979 y 1981 pasó de 196,976 a 449,645 , significando un incremento porcentual del $128.3 \%$, en tanto que el número

$\overline{5 \mathrm{Mh}} \mathrm{rx}$, op. cit. T. I: cap. 23. Aquí se encuentra un excelente desarrollo de este proceso que de por si, es característico del modo de producción capitalista. 
de quiebras de empresas pasó de 29,500 a 65,710 en el mismo período para significar un incremento del $122.70 \%{ }^{6}$ En Alemania y Japón ocurre algo similar y para 1981 el número de empresas que quebraron es de 11,580 en el primero y 17,600 en el segundo. ${ }^{7}$

Por lo que respecta al proceso de fusión o centrallzación de capitales en Estados Unidos, mientras que en 1969 se registraron 6,000 operaciones de fusión, en 1980 fueron 2,000 y 100 para $1981 .{ }^{8}$ Este descenso del proceso de centralización se explica en función de la quiebra de los más pequeños y débiles financieramente y en función de la asociación de los más grandes que, aunque más pocos, tienen un radio de acción mayor y, por tanto, modifican la estructura de la competencia dándole un carácter oligopólico. A su vez, este proceso de centralización de capitales está muy ligado a las expectativas de adquisición de importantes activos subvaluados, y de control de actividades productivas claves en ciertas ramas de la insdustria, para una reubicación de punta del capital industrial. ${ }^{9}$

\section{CONCENTRACION Y MODERNIZACION DE LA INDUSTRIA. EL PAPEL DE LA INVERSION EN INVESTIGACION Y DESARROLLO}

El crecimiento desproporcionado de la inversión en tecnología productiva en relación a la destinada al empleo de la fuerza de trabajo, es la manifestación concreta de lo que se conoce como concentración de capital en una empresa individual. Esta concentración significa una composición orgánica del capital creciente y una productividad similar. La menor incorporación de trabajo vivo en las mercancías unitarias, resultantes del mayor plusvalor obtenido de una masa de fuerza de trabajo relativamente menor y de la producción en serie, redunda en una mayor competitividad de las mismas al ser puestas en circulación en el mercado.

Esta tendencia a la concentración es ineludible por parte de aquellas empresas con intenciones y capacidad financiera para mantenerse en la competencia por los mercados, y ha obligado a transformaciones tecnológicas de

\footnotetext{
6"The Surge in bankruptcies: Is the new law responsible?" Economic Review of the Federal Reser. ve Bank of Atlanta, enero de 1982, p. 23. Citado por Burkún (S.F.):84.

7 Zaldúa (S.F.): $141(26 / \mathrm{V} / 82)$ y $135(4 / \mathrm{I} / 82)$.

8 Para 1969 y 1980 ver Le Monde, enero 14 de 1981, p. 19. Citado por Burkún, op. cit.: 72. Para 1981 ver The New York Times. Citado por Zaldus, op, cit: $119(12 / 1 \mathrm{~V} / 82)$.

${ }^{9}$ Burkun, op cit $+84-85$.
} 
gran envergadura. Sin embargo, la rápida difusión del progreso técnico y la creciente incorporación competitiva de nuevas empresas, trae consigo transformaciones tecnológicas más profundas, cuyo origen se desprende de una creciente asignación de recursos financieros a la Investigación y el Desarrollo de nueva Tecnología Productiva (en adelante IDNTP). Ahora bien, históricamente se ha venido observando que el Estado juega un papel decisivo en la orientación que se le da a la IDNTP, sobre todo en la destinada a la fabricación de armamentos; encontrándose en segundo lugar los grandes consorcios interesados en multiplicar, a través de la tecnología, sus ganancias.

Lo anterior es particularmente cierto para los gobiernos de las potencias más poderosas e industrializadas del mundo actual que, entre la segunda mitad de los sesentas y hasta mediados de los setentas, destinaron la mayoría de sus recursos a la IDNTP en las ramas de producción de los instrumentos bélicos más modernos y de mayor alcance, principalmente. Este es el caso de las industrias manufactureras de productos eléctricos y electrónicos y de aviación y espacial de los Estados Unidos en donde se aplicaron el 85.3\% de los recursos públicos en promedio; de Francia con el 92.6\% del Reino Unido con el $91.1 \%$ y; de Alemania con el $63.2 \%$. El caso de Alemania, al igual que el de Japón, es diferente en cuanto a que sus inversiones en IDNTP se canalizaron al desarrollo de nuevos medios de producción que sirvieran en la reconstrucción del aparato y la infraestructura productiva destruídos durante la guerra, como base técnica necesaria para el crecimiento industrial. El aislamiento comerical de Japón y la alta dependencia de su economía hacia el sector externo, es quizás un importante motivo para el fortalecimiento del desarrollo tecnológico de su estructura productiva y de comunicaciones. ${ }^{10}$ A mayor abundancia, se puede mencionar que el Ministerio Japonés de Industria y Comercio Internacional (MITI), está estimulando fuertemente el desarrollo tecnológico de la industria japonesa mediante la promoción y estímulos a pequeñas empresas que quieran desarrollar tecnologías prioritarias, garantizando arriba del $80 \%$ de un préstamo bancario de bajos intereses de 400,000 dólares. Estas áreas prioritarias son: biotecnología, incluyendo nuevos alimentos y drogas medicinales; nuevos materiales, tales como fibras de carbón y cerámicas; fibras ópticas para equipo de comunicación $\mathrm{y}$; microelectrónica, incluyendo robots y tecnología relacionada con computadoras. ${ }^{11}$ Esto último es muy importante en términos de modificaciones en la "estructura técnico-productiva", si se considera que la industria japonesa empleó, en 1981, 65 mil robots reprogramables. ${ }^{12}$ Sin embargo, esto no altera la tendencia ya observada y para 1980, el 240\% de los gastos mundiales en IDNTP. Se destinó a arma-

\footnotetext{
10 Fajnzylber (1980):874.

11 Grossman (1982):103.

12 Zaldua op cit.: $137(11 / 11 / 82)$.
} 
mentos; el 15\% fue para investigación básica; $15 \%$ para programas espaciales; $8 \%$ o para energía y; $70 / 0$ para salud. ${ }^{13}$

Por lo que se refiere a la inversión privada en IDNTP, esta se ha venido concentrando en la industria de productos eléctricos y electrónicos; de productos químicos $\mathrm{y}_{\mathrm{i}}$ de maquinaria. En tanto que para 1967 los empresarios de Japón, Estados Unidos, Alemania y Francia concentraron sus recursos en mayor abundancia en el campo de la química, el Reino Unido lo hizo en el campo de la electricidad y la electrónica. Para 1975, el cambio en la aplicación de recursos en IDNTP se desplazó, en el caso de los empresarios de Japón y Francia, hacia el campo de los productos eléctricos y electrónicos; en el de los Norteamericanos al de maquinaria y; los del Reino Unido lo hicieron al de la química, en donde los Alemanes se mantuvieron. Sin embargo, para todos los casos, excepción hecha del Reino Unido, la inversión privada en IDNTP cuya base fuera la electricidad y electrónica se incrementó. ${ }^{14}$ Esto es importante señalarlo, debido' a que la humanidad está asistiendo a unà revolución tecnológica de magnitudes nunca vistas que supera con creces el impacto de la Revolución Industrial, tanto en lo que se refiere a la multiplicación exponencial de la productividad industrial, como del desempleo: nos referimos a la revolución microelectrónica que está modificando desde la estructura fabril hasta la oficina tradicional, suplantando su funcionamiento mecánico por el computarizado, Esta revolución resulta mucho más importante si se considera que es la base de la transformación de la "estructura técnico-productiva" que requiere el capitalismo como una de las condiciones para salir de la crisis en que actualmente se encuentra.

Esta afirmación se basa en la observación de algunos indicadores sobre el destino de los recursos en IDNTP en primer lugar, y sobre el desarrollo de la industria electrónica en los principales países del mundo capitalista actual, en segundo lugar. Si fuera posible formalizarlo, probablemente se obtendría una alta correlación entre inversión en IDNTP; producción de la industria electrónica $y$; computarización de la producción y administración de la empresa moderna.

En la carrera por transformar la base técnica de la producción como una respuesta revolucionadora y progresista en el desarrollo del capitalismo, los industriales norteamericanos, después de tener un importante auge que los colocó como el centro dinámico del capitalismo, presentan un leve retraso respecto a los empresarios japoneses en el recambio tèenológico, lo cual les está obligando a recurrir a la asociación abierta con los centros de ense-

13 Colin Notman, The God that limps. Science and technology in the eighties, New York, Wordwatch Institute y W.W. Norton and Company, 1981. Citado por Calvillo (1982): n.p. Sobre el mismo libro ver reseña de Warman $(1982): 207-211$

${ }^{14}$ Fajnzylver, op. cit. 
ñanza superior, justificando la acción por la mutua necesidad que tienen uno de otro "en esta era de rápido paso del cambio tecnológico". 15

En efecto, el proceso de concentración de algunas empresas apoyado en los avances de la microelectrónica y su aplicación a la producción, ha sido tan ágil e impresionante que para 1980 el gasto en IDNTP realizado por algunas empresas, sobre todo en el campo de las computadoras, la industria automotriz y la industria de las telecomunicaciones, ha sido más alto que el de algunos países. Este es el caso de General Motors e IBM, cuyo gasto en conjunto es mayor que el de Italia y Suecia y, considerado particularmente. es mayor que el de Bélgica, India, Fspaña, Corea del Sur, etc. (Ver cuadro 1).

Las ventajas derivadas de la IDNTP por parte de las empresas con fuerte ritmo de concentración de capital, redundan en una mayor concentración, inclusive en el terreno de la IDNTP, ya que el constante desarrollo tecnológico exige una capacidad financiera particularmente grande para el recambio permanente de tecnología. Colin Norman menciona que en Estados Unidos son más de 10,000 las empresas que realizan investigación, el 20\% del gasto total en IDNTP es realizado por cuatro empresas: General Motors, Ford Motor Company, IBM y ATT. 16 De esto puede desprenderse, sin lugar a dudas, que en un período de recesión donde se agudice más la competencia, serán estas quienes mayores posibilidades tengan de salir adelante y en virtud de las cuales ocurra la reestructuración de la industria en el capitalismo.

\section{COMPUTADORAS Y AUTOMATIZACION DE LA PRODUCCION: SU IMPORTANCIA.}

La importancia fundamental de la introduccion de la microelectrónica a todas las ramas de la industria, radica en que abarata los elementos que componen el capital constante fijo, constante circulante y variable, contrarrestando por tanto, la disminución de la tasa de ganancia. La computadora Eniac que fuera la más grande del mundo en 1946, estaba construída, principalmente, a base de tubos de vacio. Hoy, 36 años después de que los laboratorios Bell de Estados Unidos inventaron los transitores (1947), una computadora manejada con microfichas es 300 mil veces más pequeña y 10 mil veces más velo $\%$, a la vez que mucho más barata. ${ }^{17}$ En una época en que la producción se automatiza cada vez más gracias a las computadora, el abaratamiento permanente de éstas, consideradas como capital constante fijo, favorecen la obtención de altas tasas de ganancia que facilitan la trans-

\footnotetext{
15 Business Week (1982b): 70-71.

16 Colin Norman, citado por Calvillo, op. cit

17Der Spiegel (1982b): n.p. Sobre la competencia intercapitalista en el mercado de las computadoras, Ver Business Week (1982a):48.53.
} 
CUADRO 1. Inversión en investigación y desarrollo de nueva tecnología productiva en 1980. (Millones de dólares).

\begin{tabular}{lr}
\hline República Federal Alemana & 8,847 \\
Italia & 1,656 \\
Suecia & 1,216 \\
General Motors* & 1,114 \\
IBM** $^{*}$ & 946 \\
Bélgica $^{*}$ & 764 \\
Ford Motor Company* & 748 \\
ATT*** & 619 \\
India & 420 \\
España & 262 \\
ITT* & 219 \\
Corea del Sur & 127 \\
\hline
\end{tabular}

- Automotrices

** Computadoras

*** Telecomunicaciones

FUENTE: OCDE, ONU, BUSINESS WEEK. Cirado por Rodrigo Calvillo (1982); n.p.

formación de la "estructura técnico-productiva". Se trata pues, como señaló Lester V. Colwell, profesor de Ingeniería Mecánica en la Universidad de Michigan, de "convertir" mediante la fabricación con ayuda de computadora (FAC), el "saber-hacer" de la fabricación de una tecnología "basada en la experiencia", a una tecnología "basada en la ciencia". ${ }^{18}$

La microelectrónica también ha contribuído al abaratamiento del capital variable, esto es, de la fuerza de trabajo que se convierte en cuanto que ser social, "en copartícipe del disfrute de la riqueza, (aunque sea) hasta el límite de su equivalente". 19 Con esto se quiere indicar que el valor de la fuerza de trabajo no significa tan sólo alimentos y vestidos; también requiere de satisfacciores materiales y espirituales que no siempre están a su alcance debido a lo reducido de su límite adquisitivo (salario). Por ende, el obrero y su familia aprovechan el abaratamiento de las mercancías que les satisfagan o ayuden a liberar tiempo para satísfacer sus necesidades espirituales, intelectuales, sociales, etc. Ello le permite participar materialmente de los adelantos tecnológicos de la sociedad en general, y si bien no lo eximen de una mayor explotación de su fuerza de trabajo; de una inestabilidad permanente en el empleo y; de una situación social injusta, se constituyen como medio material de su educación; esto es, de su maduración conciente como ente social, etc. Esta participación en el progreso es particularmente cierta

18 Shaiken (1981):19.

19 Ro sdolsky (1978): 233-234, nota 17. 
en el caso de algunos países donde el nivel de salario es relativamente alto y su poder adquisitivo no está muy deteriorado. Al mismo tiempo, la microelectrónica, al difundirse en las industrias productoras de medios de subsistencia, "incide de manera inmediata en desvalorizar los medios de vida del obrero". 20

Por otra parte, el efecto negativo que la microelectrónica tiene sobre el nivel de empleo, origina un crecimiento de la superpoblación relativa o ejército industrial de reserva cuyo efecto de competencia por las oportunidades de empleo, presiona sobre el nivel de salarios y sobre la productividad del obrero en activo, contribuyendo al descenso del valor de la fuerza de trabajo y a extender el tiempo de trabajo excedente, coadyuvando así a contrarrestar la disminución de la tasa de ganancia. Sobre esta situación, se prevee que durante la década de los ochentas, en Alemania Federal se perderán 2 millones de empleos debido a los avances en la mecanización del trabajo y 200 mil empleos en la industria automotriz de Estados Unidos gracias al incremento de la automatización del proceso productivo.

La tendencia a un mayor abaratamiento de los costos de producción de las mercancías gracias al creciente abaratamiento de los elementos que conforman tanto al capital constante como al variable, acompanada por un incremento sustancial de la superpoblación relativa así como de la concentración y centralización de capital en algunas ramas productivas, se ve fuertemente impulsada por la creciente automatización de la industria que se empieza a imponer sobre todo tipo de actividad económica y/o social y que, paradójicamente, se convierte en un elemento importante de la recuperación a mediano plazo, del capitalismo mundial. Esta automatización tiene como base técnica el creciente uso de las microfichas que son minitablas de 425 $\mathrm{mm}$ por lado que concentran hasta 262,144 unidades de información (es el caso del semiconductor $256 \mathrm{~K}$ RAM), cuya programación permite que se utilicen para manejar los autómatas de los billetes del metro; guiando avicnes para un seguro aterrizaje; dosificando la inyección de gasolina en los automóviles; dirigiendo los aparatos de soldadura robotizados en las cintas automáticas de las fábricas automotrices; dando la hora en los relojes de pulsera de uso común; etc. Estas minitablas concentran elementos electrónicos de conexión como diodos, transistores, circuitos y unidades de información grabados en finas muestras microscópicas. Algunas instituciones internacionales han mostrado su preocupación por el carácter dialéctico de estas miniaturas electrónicas que, a la vez que reunen un progreso tecnológico insospechado con la promesa de liberar ampliamente a la humanidad de la obligación de hacer diariamente muchos trabajos manuales y adentrarla en una época de paz social, la colocan al borde de peligrosas sacudidas sociales como nunca fueron vistas.

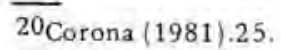


Como ya se ha mencionado, el carácter altruista del empresario no exis-. te y esa promesa de paz jamás será realidad si se espera sea otorgada por buena voluntad. Existe, por el contrario, una tendencia empresarial orientada más a crecer y concentrar tecnología productiva como base de un mayor crecimiento, que a generar empleos. Por ejemplo, la introducción de la microelectrónica en la industria relojera ha desplazado en los últimos años a $45 \mathrm{mil}$ relojeros; en el caso de la industria gráfica Alemana, la introducción de la microelectrónica, a la vez que elevó su productividad en $43.5 \%$ o entre 1970 y 1977, disminuyó el nivel de empleo en 21.3\% o. $^{21}$ Gamble y Walton, refiriéndose al caso de Gran Bretaña, mencionan que hubo una época en que el pleno empleo era un elemento indispensable en los planteamientos de todas las organizaciones políticas; ahora ha desaparecido esa garantía, no porque el pleno empleo sea imposible de lograr, sino porque no es redituable. ${ }^{2} 2$ En agosto de 1982 la tasa de desempleo ascendió al 13.8\% de la PEA para significar el más alto nivel en toda la historia de Gran Bretaña. Sin embargo, las políticas monetaristas de Margaret Thatcher han logrado reducir la inflación y la tasa de interés, y mejorado relativamente la competitividad de las mercancías británicas en el mercado mundial. ${ }^{23}$

Ahora bien, el impetuoso desarrollo observado en el campo de las computadoras deviene en una de sus vertientes, de la creciente incorporación de estas a la producción, trayendo consigo la automatización del proceso productivo y el consiguiente desplazamiento de fuerza de trabajo. En la misma industria electrónica, se piensa que las microcomputadoras eliminarán más empleo ya que están produciendo nuevos elementos electrónicos sin el auxilio humano. Sobre el particular opina Harley Shaiken, Sociólogo del Massachusets Institute Technologic, que la mano invisible reguladora del mercado de trabajo que tanto reivindican las políticas neoclásicas de empleo, ahora es artrítica e incluso paralítica, ya que el parecer no hay modo de esquivar el ataque de las microcomputadoras y de los robots industriales. ${ }^{24}$

En los albores de ésta década se observó un concenso entre OCDE, Comisión Brandt, ONU y Ministerio de Relaciones Exteriores de la Gran Bretaña, "sobre la necesidad de restructurar la industria (. . .) sustituyendo ramas intensivas en mano de obra por ramas intensivas en capital". $25^{\circ}$ A fin de otorgarle a las naciones Europeas un lugar importante en la conformación de la nueva división internacional del trabajo. En el caso de Gran Bretaña, los empresarios necesitan correr en la carrera por automatizar la producción a fin de no rezagarse en la competencia intercapitalista mundial.

21Der Spiegel, op. cit.

22 Gamble y Walton (1979): 22.

23 Uno más Uno, agosto 26 de 1982 .

24 Der Spiegel, op. cit.

25 FitzGerald (1981):412. 
Para ello el gobierno Inglés ha ofrecido subsidios del 25\% de los gastos resultantes de la aplicación de robots a la producción, y son dos las empresas adentradas ya en tal proyecto ${ }^{26}$ En esta carrera se observa la asociación internacional de capitales entre Sykes Group a través de su subsidiaria Woodsome Park Computer con el especialista Japonés en robots Dainichi Kiko y la conformación de la nueva empresa Dainichi Sykes, que con un presupuesto inicial de 4 millones de libras, planea producir de 10 a 15 robots mensuales. El objetivo de los ingleses con esta asociación es acortar el camino que en IDNTP tienen ya recorrido los Japoneses. Para estos últimos, el objetivo principal consiste en penetrar el mercado Inglés de los robots. Como dato adicional, el 30\% de los empleados de la empresa Dainichi Sykes está ocupado en el laboratorio de IDNTP. Otro casu similar es el del conglomerado 600 Group con Fujitsu Fanuc, sólo que en este caso existe el obstáculo de que la empresa Alemana Siemens tiene los derechos pagados para utilizar la tecnología Fanuc en Europa y por ende ofrece la producción de robots que Ia subsidiaria Hydro Machine Tools requiere. Para evitar el intermediario, ésta última está planteando un renegociamiento de los acuerdos sobre derechos tecnológicos con Fanuc. ${ }^{27}$

La utilización intensiva de capital en las ramas industriales donde la competencia es más intensa, actualmente sólo es rentable mediante la automatización de la producción por medio de robots. El auge de esta tecnología se originó en Japón en 1967 y se extiende 5 años después a los Estados Unidos donde dos empresas controlan el 70\% del mercado. En la actualidad, de las 280 empresas que producen robots en el mundo, el $53.6 \%$ o son Japoneses y su característica principal es que no se dedican tan sólo a la producción de robots. ${ }^{28}$ En efecto, muchas de las corporaciones Japonesas han instalado pequeñas subsidiarias dedicadas a actividades de alta tecnología, especialmente la de sistemas lógicos de computación (software). ${ }^{29}$

No en balde los mayores adelantos tecnológicos en lo que a automatización de la producción se refiere, se dan en el campo de la producción de sistemas lógicos computacionales o producción "software", y ya se implementan algunos sistemas de este tipo en el mundo. Por cjemplo, con el Sistema de Manufactura Flexible (FMS) la producción de componentes metálicos es totalmente automática y sólo las operaciones de carga y descarga, aparte de la supervisión y programación, se realiza con actividad humana. Actualmente existen 75 instalaciones FMS operando a escala mundial en Japón, Alemania, Estados Unidos y Gran Bretaña; la ventaja de su utiliza-

26 The Economist (1981): 69-70.

27 Ibid.

28 Gray (1982) 33.

29 Grossman, op, cit 
ción en un taller que produce 1.8 millones de libras, radica en que se pueden lograr ahorros hasta de 160 mil libras o una utilidad extra del 9\%o. Por su parte, el sistema NEL es un enfoque modular de la producción en que pueden agregarse máquinas y placas giratorias adicionales que están manejadas y adecuadamente asociadas en una computadora de consola, y las instrucciones sobre la fabricación de un producto con determinadas características, se envían al control en el centro de producción. ${ }^{30}$

Estos sistemas trabajan con robots industriales que son generalmente estacionarios y en ciertas versiones lucen como un gigantesco brazo y muñeca de metal emplazados sobre un pedestal que pueden soldar carrocerías, cargar máquinas e incluso ensamblar partes complicadas. Cuando se requieren cambios en el producto, basta con dictar nuevas instrucciones al sistema de control basado en el microprocesador, para que el robot se adapte a las nuevas tareas. ${ }^{31}$

Estos robtos, a la vez que están reduciendo los requerimientos de fuerza de trabajo en el proceso productivo, eliminando con ello las posibilidades de interrupción debido a conflictos laborales, están elevando la productividad y la eficiencia, reduciendo los costos por concepto de fuerza de trabajo y elevando el grado de explotación de la que se encuentra empleada. Un robot que trabaje pintando en la industria automotríz puede ahorrar hasta un 20 o $30 \%$ o de la pintura que utiliza un operario humano. ${ }^{32}$ y cuesta aproximadamente 4.80 dólares por hora, una vez que su precio de 40 mil dólares y demás gastos de mantenimiento se distribuyen a lo largo de 8 años de vida útil durante 2 turnos de 8 horas diarias. ${ }^{33}$ Un ejemplo de éste tipo de economías se observa en la fábrica de neumáticos Good Year, en Estados Unidos, donde en cada uno de los dos turnos había 2 mujeres que transportaban pesadas ruedas de un lado a otro. Al ser sustituídas por un robot de 23,000 dólares, la Good Year ha economizado 28,000 dólares en salarios durante $1979,{ }^{34}$

En la carrera por automatizar la producción por medio de robots, las empresas de Japón producen el 85.1 de ellos y utilizan el 89.5 de los mismos a nivel mundial (ver cuadro 2 ). $\mathrm{Y}$ aunque la tendencia de automatización de la producción es observable para todas las ramas industriales claves de la economía, por lo pronto ésta se está concentrando con mayor énfasis en las insdustrias automotriz, eléctrica y electrónica. En los Estados Unidos están siendo utilizados por General Motors y Ford Motor Company; en

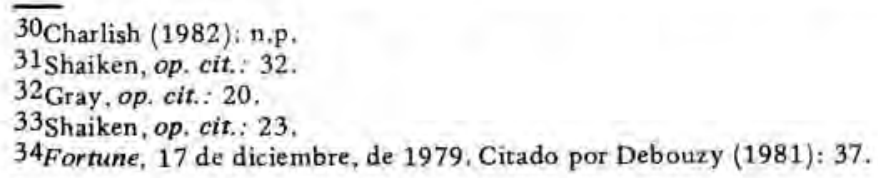


CUADRO 2. Industria robótica de 1980.

\begin{tabular}{lcc}
\hline PAIS & PRODUCCION & UTILIZACION \\
Japón & 12,000 & 47,000 \\
Estados Unidos & 1,367 & 3,000 \\
Alemania & $*$ & 800 \\
Francia & 50 & $-*-$ \\
Gran Bretaña & $*$ & 360 \\
Suecia & 460 & 1,000 \\
Noruega & 140 & 180 \\
Finlandia & 80 & 120 \\
Dinamarca & $-*$ & 70 \\
\hline
\end{tabular}

FUENTE; Federación Internacional de Sindicatos Metal Mecánicos, Citado por Carrillo, Jorge., La industria maquiladora en la frontera México-Estados Unidos y la internacionalización del capital, Denver, Colorado, Association for Borderlands Scholars, Annual meetings (ponencia), 22-23 de abril de 1982 , p. 8.

Italia por Fiat; en Alemania por Volkswagen y; en Japón por Nissan, Toyota y Kogyo's en lo que se refiere a la rama automotriz. ${ }^{35}$ Esta última empresa japonesa, hace seis años llegó casi a la quiebra, siendo salvada por un importante banco a cambio de una automatización total de la producción. En este caso los miles de trabajadores en las cintas automáticas no fueron, despedidos, sino que siguieron trabajando para la empresa como vendedores de autos distribuidos por todo el Japón. ${ }^{36}$ En la industria japonesa, la utilización de robots se aplica en la tendencia descrita, con la salvedad de que en 1980 la mayor utilización de robots fue en la industria electrónica, sin que en las demás ramas se observen incrementos sustanciales (Ver cuadro 3 ).

A diferencia de la automatización primaria en que la máquina sustituye al trabajador manual no calificado, los robots sustituyen obreros calificados y experimentados, llegándose a extremos tales como el de Fujitsu Fanuc que en 1982 abrió una fábrica de 50 millones de dólares cón solamente 30 obreros que producen 10 mil ejes de motor mensualmente. ${ }^{37}$ En realidad, como señalaron los ingenieros de la General Motors, se ha decidido conservar a "seres humano (...) hasta que los sistemas táctiles y de visión se perfeccionen y sean económicamente accesibles". 38 Luego enton-

\footnotetext{
35Para E.U. ver Shaiken, op. cit.: 24 y 28 y Baranson (1981): 156.; para Italia y Alemania ver Rigacci (1981): 8-9; Para Japón ver Gray, op, cit.: 27 y Baranson, op. cit.: 156.

36Der Spiegel (1982a): n.p. También ver Baranson, op, cit.: 156-157.

37 Gray, op. cit.: 38 .

38 American Machinist. "Robot trend at General Mo tors", august 1979, p. 71. Citado por Shaiken, op. cit: 24 . Se piensa que con el tiempo, las computadoras serán mucho más rápidas y utilizarán componentes menos complicados. Conaway (1983): n.p.
} 
CUADRO 3. Porcentaje de robots comprados en la industria japonesa por rama productiva.

\begin{tabular}{lrrrrr}
\hline Rama industrial & 1976 & 1977 & 1978 & 1979 & 1980 \\
Automotriz & 30 & 34 & 39 & 38 & 29 \\
Electrónica y maquinaria eléctrica & 21 & 23 & 24 & 18 & 36 \\
Maquinaria de precisión & 1 & 1 & 1 & 3 & 2 \\
Maquinaria de construcción & 1 & 1 & 1 & 1 & 1 \\
Maquinaria de minería & 5 & 6 & 4 & 3 & 4 \\
Otra maquinaria & 2 & 2 & - & 1 & 1 \\
Bicicletas y vehículos industriales & 2 & 2 & 1 & 1 & -10 \\
Productos y plásticos moldeados & 13 & 10 & 10 & 11 & 10 \\
Productos químicos, petroleros y carboniferos & 2 & 1 & 1 & - & 1 \\
Cerámica & 1 & - & - & - & 1 \\
Hierro y acero & 6 & 6 & 3 & 4 & 3 \\
Metales no ferrosos & 2 & 2 & 2 & 2 & 3 \\
Máquina y turbinas de metal y calentadorcs & 7 & 3 & 8 & 9 & 5 \\
Textiles & 1 & 1 & - & 3 & 1 \\
Otros & 4 & 4 & 4 & 4 & 2 \\
Exportaciones & 2 & 4 & 3 & 2 & 3 \\
\hline
\end{tabular}

FUENTE: Kanji Yonemoto, Japanese (ndustrial Robot Association (JIRA). Cirado por Gray, op. cit. 24

ces, la automatización totalizante de la producción es una cuestión de tiempo, definida tan sólo por el ritmo de progreso técnico y la rentabilidad de su implementación en el contexto de la competencia itercapitalista mundial.

Pero, ca dónde lleva todo esto?

La respuesta ha venido siendo tratada a lo largo del trabajo. El capitalismo sólo puede alargar su permanencia gracias a su carácter revolucionario, competitivo a inhumano. La reestructuración de las condiciones de producción a través de la modificación de los procesos de trabajo sobre la base de la producción automatizada de la cual los robots son sólo un aspecto, permitirá, una vez recuperados los elevados montos de inversión que implica el recambio tecnológico de la "estructura técnico productiva", la extensión del tiempo de trabajo excedentario de la fuerza de trabajo a través de la intensificación de su explotación, a la vez que una mayor competitividad de las mercancías en términos de la disminución del valor unitario de las mismas. Sin embargo, en esta tendencia de reestructuración son los empresarios norteamericanos quienes, guiados por una filosofía empresarial que se vió superada por la de los empresarios japoneses, la cual es motivada por condiciones materiales de reproducción diferentes, se muestran ya rezagados en el ritmo de innovación tecnológica que exige el cambio. Esto es menos cierto para los empresarios de la industria eléctrica y electrónica que aún en estos tiempos de crisis global de la ganancia industrial, mantie- 
nen márgenes positivos de ganancia al poder competir en términos de productividad y eficiencia con los empresarios japoneses y europeos en el creciente mercado que significa la modernización de la estructura productiva del sector industrial norteamericano productor de bienes de consumo para asalariados y capitalistas, que es hoy el sector dinámico de la industria norteamericana. Según indicadores de ganancias acumuladas para los primeros 9 meses de 1981 y 1982 , en tanto que la producción de medios de producción vió descender sus márgenes de ganancia en $18.4 \%$ o, la dedicada a la producción de bienes de consumo se incrementó en aproximadamente 1 ó $2 \%$ o si se considera la producción eléctrica y electrónica para consumo doméstico. Este sector de la producción industrial norteamericana se ha visto estimulado también por la creciente automatización de las actividades administrativas, financieras y bancarias, y por la creciente automatización e integración vertical y horizontal de las empresas productores de computadoras $^{39}$ que han podido mantener, gracias a ello, posiciones crecientemente competitivas dentro del mercado, y con ello, márgenes permanentes positivos de ganancia. (Ver cuadro 4).

\section{PERSPECTIVAS DE LA AUTOMATIZACION}

La automatización de la producción por computadoras que controlen robots numéricamente controlados, a saber lo más adelantado del ramo, tiende a generar mayor automatización y a replantear los términos de competencia entre grandes y pequeños. Se estima que para 1984 el $40 \%$ de los robots estarán instalados en industrias relativamente pequeñas, lo que significaría una tendencia creciente hacia industria con uso intensivo de capital como la de manufacturas de precisión; la maquinaria especial, etc., que hoy se encuentran tan deprimidas y que, por tanto, requieren ser restructuradas.

Japón se está planteando para 1992 el dominio tecnológico con una computadora que pueda pensar, contar y comunicarse directamente con los humanos sin necesidad de cintas, teclados y programadores; lo mismo se pretende con los robots y para ello el Ministerio Japonés de Industria y Comercio Internacional (MITI), se ha propuesto elaborar un proyecto de investigación conjunto por siete años con una inversión de 140 millones de dólares, y donde varias importantes compañías están involucradas; entre ellas, Hitachi ya tiene 500 investigadores trabajando. ${ }^{40}$

Todo esto significa un mayor abaratamiento de los elementos del capital constante fijo y también del capital variable, debido al incremento del

39 Business Week (1982): n.p.

${ }^{40}$ Sexton (1982): n.p. 
CUADRO 4. Ganancias industriales por sector de producción en Estados Unidos 1981-1982. (Millones de dólares).

\begin{tabular}{|c|c|c|c|}
\hline & $\begin{array}{c}\text { Enero-Sept. } \\
1981\end{array}$ & $\begin{array}{l}\text { Enero-Sept. } \\
1982\end{array}$ & \\
\hline \multicolumn{4}{|l|}{ INDUSTRIAS } \\
\hline $\begin{array}{l}\text { Industrias Productoras de Medios de } \\
\text { Producción: I }\end{array}$ & $12,580.7$ & $10,259.9$ & -18.4 \\
\hline Herramientas e Instrumentales & 274.0 & $194.3^{\circ}$ & -41.0 \\
\hline Químicas & $4,137.9$ & $3,207.7$ & -29.0 \\
\hline Conglomerados & $2,901.4$ & $2,284.6$ & -27.0 \\
\hline Recipientes & 336.3 & 206.3 & -63.0 \\
\hline Maquinaria en General & 554.0 & 335.1 & -56.0 \\
\hline Instrumentos de Precisión & 265.8 & 235.2 & -13.0 \\
\hline Maquinaria Especial & 297.6 & 162.6 & -83.0 \\
\hline Acero & $-1,125.0$ & 978.0 & 13.0 \\
\hline Neumáticos y productos de hule & 412.4 & 292.5 & -+1.0 \\
\hline Productos forestales y de papel & $1,580.4$ & $1,097.5$ & -44.0 \\
\hline Eléctrica y Electrónica & $2,945.9$ & $3,202.1$ & 8.0 \\
\hline \multicolumn{4}{|l|}{ Industrias Productoras de Bienes } \\
\hline Salario y Bienes de Lujo: II & $18,781.6$ & $18,858.9$ & 4 \\
\hline Bebidas & 587.3 & 707.6 & 17.0 \\
\hline Drogas (uso médico) & $3,039.5$ & $3,662.0$ & 17.0 \\
\hline Procesamiento de alimentos & $2,827.2$ & $2,667.2$ & -6.0 \\
\hline Industrias de Tiempo Libre & $1,309.3$ & $1,283.6$ & -2.0 \\
\hline Manufacturas de Misccláncas & $2,176.3$ & $1,741.0$ & -25 \\
\hline Textiles y Ropa & 810.4 & 554.4 & -16.0 \\
\hline Tabaco & $1,381.4$ & $1,625.2$ & 15.0 \\
\hline Productos de cuidado personal & $1,356.2$ & $1,356.2$ & 0.0 \\
\hline Aeroespacial & $1,063.3$ & 850.6 & -25.0 \\
\hline Automotriz & $-{ }^{*}-$ & 187.5 & $-*$ \\
\hline Equipo de Oficina: Computadoras & $4,230.7$ & $4,598.6$ & 8.0 \\
\hline GANANCIA SIECTOR INIDUSIRIAL. & $31,362.2$ & $29,118.8$ & -7.2 \\
\hline
\end{tabular}

FUENTE: Elaborado con datos de Standar and Poors Co mpustat Service fnc. Corporate Scorcboard. Third-duartes 1982. profits for 875 companies, en Business Week. New York. MeCiraw Hill Publications. November 15. 1982. IPp. 51-76.

desempleo y a la mayor oferta de fuerza de trabajo que redunda directa o indirectamente en un incremento de la tasa de ganancia. En estos términos, es muy probable que los grupos de trabajadores con mayor nivel de organización puedan lograr la reducción de la jornada de trabajo en vez del despido de trabajadores por introducción de tecnología. Esto ha sucedido ya en Inglaterra donde la sección de oficinistas del sindicato de la industria gráfi- 
ca (NATSOPA) "obtuvo una semana de 31 horas y media, 6 semanas de vacaciones pagadas, licencia de paternidad y maternidad pagada y otros beneficios que reducen la jornada de trabajo con paga completa". ${ }^{41}$

En tanto esta situación no se generalice por la propia iniciativa de los trabajadores, el desempleo tiende a ser una de las características predominantes de la sociedad moderna, a no ser porque el movimiento mismo del capitalismo tenderá a verse frenado en la medida que la demanda se constriña, y seguro es, se buscarán nuevas formas de emplear productivamente a la población. En la medida que se retarde la implementación de estas nuevas formas que con seguridad no serán producto altruista de los empresarios, se incrementarán las posibilidades de una respuesta de la población trabajadora, tendiente sin lugar a dudas, a modificar la estructura de las relaciones sociales de producción capitalistas. De esta forma, el principio de la reestructuración capitalista materializado en "la extrema degradación de la fuerza de trabajo en la realidad de la fábrica" coincidiría, por su propia dialéctica, con el principio de liberación del proletariado" 42 en el afán de humanizar la producción y distribución de la riqueza.

\footnotetext{
${ }^{41}$ Shaiken, op. cit.: 32 . Sobre la actividad del movimiento obreto norteamericano ante el proceso de transformación de la "estructura técnico-productiva", ver Debouzy, op. cit.

42 Bolchini, op. cit.: 12 .
} 


\section{Alejandro Mungaray}

\section{BIB LIOGRAFIA}

AGLIETTA, Michel. (1979), Regulación y crisis del capitalismo, México, D.F., S. XXI. eds, 2a. ed., 344 pp.

BARANSON, Jack. (1981); The Japanese challenge to U.S. industry Lexington, Mass., D. C. Heath \& Company, $188 \mathrm{pp}$.

BUSINESS WEEK (1982a), "The comming shakeout in Personal computer", New York, McGraw Hill Publications, November 22, pp. 48-53.

-_- (1982b), "Corporation Bet on Campus R \& D", New York, McGraw Hill Publications, december 20, pp. $70-71$.

BOLCHINI, Piero. (1980), "Karl Marx y la historia de la Técnica", en Karl Marx, capital y tecnología. Manuscritos inéditos (1861-1863), México, D.F., Ed. Terranova, pp. 9-23.

BURKUN, Mario. (S.F.), "Fusiones y Concentración del capital en Estados Unidos (1981)", en Mapa Econó mico Internacional (Fecha de cierre: 30 de julio de 1982), México, D.F., CIDE, pp. 71-88.

CALVILLO, Rodrigo (1982), "El Dios que cojea", en Excélsior, México, D.F., 23 de febrero.

CASTELLS, Ma nuel (1981), La teoría marxista de las crisis económicas y las transformaciones del capitalismo, México, D.F., S. XXI, eds. 2a.ed.,145 pp.

CONAWAY, James (1983), "Computadoras menos complicadas y más rápidas", en Excélsior, México, D.F., 31 de mayo.

CORONA Treviño, Leonel (1981), Tipificación de los procesos de Trabajo, México, D.F., 2o. Seminario Internacional sobre Crisis, Nuevas Techologías y Procesos de Trabajo (ponencia 3), DEPFE y DEPFCPyS, UNAM, 20 al 00 de juio, 36 pp.

DEBOUZY, Marianne. (1981), "Los sindicatos americanos frente a la invasión tecnológica", en Transición, Madrid, Ed. Blume, febrero No. 24, pp. 34-40.

DER SPIEGEL (1982a), "Arrasará los empleos: Club de Roma", en Excélsior, México, D. F., 15 de marzo. (1982b), "Inevitable reducir la jornada laboral", en Excélsior, México, D. F., 16 de marzo.

ECONOMIST, THE (1981). "British robots. Japanese style", New York-London, vol. 280, Num. 7204, September 26 pp. $69-70$.

FAJNZYLBER, Fernando (1980), "Industrialización, bienes de capital y empleo en las economías avanzadas", en Comercio Exterior, México, D.F., BNCE, Vol. 30, Núm. 8, agosto, pp. $867-880$

FITZGERALD, E.V.K. (1981), "La reestructuración de las economías de México y los Estados Unidos: una visión europea”, en C.W. Reynolds y C. Tello (Eds.), Las relaciones México-Estados Unidos, México, D.F., F.C.E. (Lecturas del Fondo 43), pp. 410-425.

GAMB LE, Andrew y Walton, Paul. (1979), El capitalismo en crisis, la inflación y el Estado, México, D. F., S. XXI. eds. , 301 pp.

GRAY. Christopher S. (1982), "Japan. Corporate Strategies for the 80's", en Business Week, New York, McGraw Hill Publications, November 29, pp. 18-44.

GROSSMAN, Robin. (1982), "Why venture capital won't work for the Japanese?", en Business Weeh, New York, McGraw Hill Publications, November 29, p. 103.

MARX, Carlos. (1978), El Capital. Critica de la Economia Politica, México, D.F., F.C.E., 13 reimp. 3 vols.

RIGACCI, Giani (1981), Il Caso Italia, México, D.F., 2o. Seminario Internacional sobre Crisis, Nuevas Tecnologías y Procesos de Trabajo (ponencia 10), DEPFE y DEPFCPyS, UNAM, 20 al 30 de julio, 20 pp.

ROSDOLSKY, Roman, (1978), Génesis y estructura de El Capital de Marx (estudios sobre los Grundrisse), México, D.F., S. XXI. eds., 630 pp.

SEXTON, William (1982), "Superará el liderazgo de E.U. en 92", en Excélsior, México, D.F., 16 de marzo

SHAIKEN, Harley (1981), "Computadoras y relaciones de poder en la fábrica", en Cuademos Politicos, México, D.F., Ed. ERA, octubre-diciembre, Núm. 30, pp. 7-32.

WARMAN, José (1982) Nota bibliográfica sobre "The God that limps. Science and technology in the eighties, de Colin Norman" en Ciencia y Desarrollo, México, D.F., CONACYT, julio-agosto, núm. 45, año VIII. pp. 207-211.

WEISSKOPF, Thomas E. (1980), "Teoría marxista de la crisis y la tasa de ganancia en la economía nor teamericana de la posguerra", en Cuadernos Semestrales. Estados Unidos: una perspectiva latinoamericana, México, D.F., CIDE, 1er. sem. Núm. 7, pp. 55-110.

ZALDUA, Josexto. (S.F.), "Cronología (julio de 1981-junio de 1982)", en Mapa Económico Internacional (Fecha de cierre: 30 de julio de 1982), México, D.F., CIDE, pp. 131-142. 